\title{
Combining ability in some genotypes of bread wheat (Triticum aestivum L.) under different sowing dates
}

\author{
M.A. Ali ${ }^{1}$, M.S. Hassan ${ }^{1 *}$ and I.A. Ali $^{2}$
}

${ }^{1}$ Department of Agronomy, Faculty of Agriculture, South Valley University, Qena, Egypt.

${ }^{2}$ Qena Directorate of Agriculture, Qena, Egypt.

\begin{abstract}
Sixty four genotypes ( $28 \mathrm{~F}_{1}$ 's, $28 \mathrm{~F}_{2}$-populations and their 8 parents) were evaluated for days to heading, plant height, 100-grain weight and grain yield/plant under two sowing dates (Nov., 22 ${ }^{\text {nd }}$, normal sowing date and Dec., $30^{\text {th }}$, late sowing date) in 2017/2018 growing season at the Exp. Farm of South Valley Uni., Qena, Egypt. The differences among parents, F1's, F2-populations and parents vs crosses were highly significant at both planting dates for all studied traits. Delaying sowing date reduced performance all studied traits. The parents; $\mathrm{P}_{3}, \mathrm{P}_{4}, \mathrm{P}_{6}$ and $\mathrm{P}_{8}$ and the crosses; $\mathrm{P}_{1} \times \mathrm{P}_{3}, \mathrm{P}_{1} \times$ $\mathrm{P}_{6}, \mathrm{P}_{1} \times \mathrm{P}_{8}, \mathrm{P}_{2} \times \mathrm{P}_{3}, \mathrm{P}_{2} \times \mathrm{P}_{5}$, and $\mathrm{P}_{3} \times \mathrm{P}_{8}$ displayed HSI and $\mathrm{S}$ less than one and they were relatively heat tolerant and less sensitive to heat of $F_{1}$ and $F_{2}$-generations. Highly significant differences due to general (GCA) and specific (SCA) combining abilities were detected for all studied traits. The ratio of GCA to SCA was less than one for all studied traits, indicating that the major effect in the control of these traits was by non-additive gene action, except plant height. The parent $\mathrm{P}_{3}$ was considered as the best combiner for heading, plant height and grain weight, but the parent $\mathrm{P}_{2}$ was considered as the best combiner of $\mathrm{F}_{1}$ 's and $\mathrm{F}_{2}$-generations for 100-grain weight and grain yield/plant under both dates. The cross $\mathrm{P}_{2} \times \mathrm{P}_{8}$ could be considered as the best combination of $\mathrm{F}_{1}$ 's and $\mathrm{F}_{2}$-generations for all studied traits under both planting dates.
\end{abstract}

Key words: Bread wheat; Combining ability; Sowing dates

\section{Introduction}

Wheat is considered as the first strategic food crop all over the world as well as in Egypt. Exposure wheat plants to various stresses during the growing season caused reductions in crop yield. Wheat cultivars that can with stand abiotic stresses particularly terminal heat tolerance will be able to fulfill the food demand in coming years (Iqbal et al, 2017).

Therefore, development of new improved wheat cultivars with high genetic potential for yield under stress environment

*Corresponding author: M. S. Hassan

elsharany@yahoo.com

Received: September 8, 2020;

Accepted: October 3, 2020;

Published: October 18, 2020. has become a major objective in wheat breeding programs. For improvement of wheat yield, study of the genetic structure and plant behavior under heat stress condition is a great important. Also, knowledge of general and specific combining ability along with the mode of gene action in the available breeding material is very important to start the effective wheat breeding programs (Kumar et al; 2017).

Hayman (1954 a and b), Jinks (1954) and Griffing (1956) stated that half diallel mating as an effective strategy to evaluate genotypes used as parents to study combining ability effects in order to select suitable parents for developing new cultivars. 
General and specific combining ability effects for yield and its components in bread wheat were reported by several workers (Dueby et al, 2001; Wahid et al, 2007; Ahmed and Mohamed, 2009; Kapoor et al, 2011; Ankita et al, 2012 and Kumar et al, 2017). Furthermore, each of Ali (2006), Farhad et al (2011), Yao et al (2011), Ezatollah et al (2013), Hassan (2015), Khaled and Abd ElDayem (2016) and Kumar (2017) found that each of additive and non-additive gene effects were equal in their importance in the control of most traits such as, plant height, grain yield, number of spikes/plant, number of grain/spike and 100-grain weight. Additive and non-additive gene effects were more important in controlling the genetic system for plant height, grain yield, number of spikes/plant, number of grain/spike and 100grain weight (Hamada and Tawfeleis (2001), El-Sayed (2004), Abdel-Nour, Nadya, et al (2009), Moussa (2010) and El-Awady, Wafaa (2011). The main objectives of the present study were: 1 - To determine the general and specific combining abilities in bread wheat genotypes under heat stress condition. 2- To identify the best combiners and its combinations under heat stress conditions.

\section{Materials and Methods}

The current research was performed at the Experimental Farm of Faculty of Agric., South Valley Univ., Qena, Egypt during 2015/2016 - 2017/2018 growing seasons. The parental materials used in this study were eight genotypes of bread wheat (Tritium aestivum L.) namely; Line158 $\left(\mathrm{P}_{1}\right)$, Shamiss$3\left(\mathrm{P}_{2}\right)$, Gemmiza-11 $\left(\mathrm{P}_{3}\right)$, Sakha-93 $\left(\mathrm{P}_{4}\right)$, Misr-2 $\left(\mathrm{P}_{5}\right)$, Shandweel-1 $\left(\mathrm{P}_{6}\right)$, Sids-1 $\left(\mathrm{P}_{7}\right)$ and Giza-168 $\left(\mathrm{P}_{8}\right)$ representing a wide range of genetic diversity for several traits were selected for this study.

In 2015/2016 growing season, the eight parents were used in a half diallel cross mating design and seeds of $28 \mathrm{~F}_{1}$ 's were produced. In 2016/2017 growing season, ten seeds per each cross were sown to produce seeds of generation.

In 2017/2018 growing season, all genotypes (the parents, $\mathrm{F}_{1}$-crosses and $\mathrm{F}_{2}$ populations) were evaluated under to sowing dates; normal $\left(22^{\text {nd }}\right.$ of Nov.) and late $\left(30^{\text {th }}\right.$ of Dec.) in two separate traits. In each trail, the genotypes were grown in a randomized complete block design (RCBD) with three replicates. Each replicate included one row, 3 $\mathrm{m}$, long and $30 \mathrm{~cm}$ apart with $10 \mathrm{~cm}$ between plants within row. All recommended cultural practices were applied.

Fifteen guarded plants for each genotype were taken randomly from each replicate to recording observation on days to heading, plant height, 100-grain weight and grain yield/plant. Some physical and chemical properties of the soil of the experimental site are shown in Table 1. Minimum and maximum and mean daily temperatures at Qena of 2017/2018 season are shown in Table 2.

\section{Statistical analysis}

The analysis of variance for randomized complete block design for each experiment was done according to Snedecor and Cochran (1967). The diallel analysis was conducted according to Griffing (1956) method II model I (without reciprocal cross). Heat susceptibility index (HSI) was calculated according to Fischer and Maurer (1978). In addition, the sensitivity of any genotype or cross was estimated according to Falconer (1990).

\section{Results and Discussion}

The results obtained from the analysis of variance for all studied traits of 64 genotypes ( 8 parents, $28 \mathrm{~F}_{1}$ hybrids and 28 
$\mathrm{F}_{2}$-progenies) under normal and late planting dates (Table 3) revealed highly significant differences among genotypes for all studied traits indicating that the importance of evaluation these traits over environments. The differences among parents, $\mathrm{F}_{1}$-crosses and $\mathrm{F}_{2}$ generations were highly significant for all studied traits under both sowing dates, this indicates that a wide diversity was found among the parental materials used in the present study. In addition, mean squares due to parents vs. crosses were highly significant for all studied traits, reflecting the average heterotic effect for these traits.

\section{Days to heading date (day):}

The average of days to heading for parents was 85.33 days at normal date, while it was reduced to 75.25 days under late date. The earliest parents were Sakha-93 $\left(\mathrm{P}_{4}\right)$ and Gemmiza-11 $\left(\mathrm{P}_{3}\right)$ under normal and late sowing dates which headed after 81.33 and 70.33 days from sowing date, respectively. While, the latest parents; Shamiss-3 $\left(\mathrm{P}_{2}\right)$ and Sids-1 $\left(\mathrm{P}_{7}\right)$ were headed after 89.33 and 81.00 days from sowing under normal and late sowing dates, respectively (Table 4).

The earliest $\mathrm{F}_{1}$-hybrids were Sakha- $93 \times$ Sids-1 and Sakha-93 $\times$ Giza-168 under normal date and Shamiss-3 $\times$ Gemmiza-11, Gemmiza-11 $\times$ Sakha-93 and Gemmiza-11 $\times$ Giza-168 under late date headed after 77.67 and 68.67 days from sowing, respectively. While the latest F1-hybrid was Shamiss-3 $\times$ Misr-2 that headed after 85.00 and 72.33 days from sowing at normal and late dates, respectively. Late planting date reduced days to heading over all F1-hybrids from 81.11 (normal date) to 71.05 days (late date), (Table 4).

The average days to heading for $\mathrm{F}_{2}-$ generations ranged from 73.00 for Line $158 \times$
Shandweel-1 to 80.00 for Line158 $\times$ Misr-2 with an average of 75.35 days under normal date and from 68.33 for Sakha-93 $\times$ Giza168 to 72.33 for Shandweel-1 $\times$ Sids- 1 with an average of 70.40 days under late planting date (Table 4).

In general, late planting date decreased average days to heading by 12.10 and $9.97 \%$ over all genotypes compared with normal date for $\mathrm{F}_{1}$ 's and $\mathrm{F}_{2}$-populations, respectively. However, days to heading of the $\mathrm{F}_{2}$-populations were affected greatly than the $\mathrm{F}_{1}$-hybrids by stress condition. Most of the $\mathrm{F}_{2}$ populations of parent 4 (Sakha-93) headed earlier than the population of the other parents. These results are also in line with those obtained by Hassan (2015), Hassan (2016), Jaiswal et al (2017), Asmaa et al (2018), Sharma et al (2019) and Bajaniya et al (2019).

\section{Plant height (cm):}

Concerning plant height, the average of parents ranged from 56.91 for Shamiss-3 $\left(\mathrm{P}_{2}\right)$ and 47.96 for Sakha-93 $\left(\mathrm{P}_{4}\right)$ to 75.64 and 63.11 for Sids-1 $\left(\mathrm{P}_{7}\right)$ with an average of 66.57 and $56.15 \mathrm{~cm}$, under normal and late planting dates, respectively (Table 4).

Mean of plant height for $F_{1}$-hybrids showed that the tallest plants were Gemmiza$11 \times$ Sids- 1 by $80.78 \mathrm{~cm}$, and Misr- $2 \times$ Sids1 by $59.53 \mathrm{~cm}$., but the shortest plants were Line $158 \times$ Sakha-93 by $63.13 \mathrm{~cm}$. and Sakha-93× Giza-168 by $48.64 \mathrm{~cm}$. under normal and late planting dates, respectively (Table 4). The average of plant height for $\mathrm{F}_{2}$ generations ranged from 60.71 and $49.53 \mathrm{~cm}$. for Shamiss-3 $\times$ Sakha-93 to $80.07 \mathrm{~cm}$. for Sids- $1 \times$ Giza-168 and $66.21 \mathrm{~cm}$. for Line 158 $\times$ Gemmiza-11 with an average of 71.43 and $57.50 \mathrm{~cm}$. under normal and late planting dates, respectively. The reduction was about 
$19.58 \%$ and $17.65 \%$ in plant height for $\mathrm{F}_{1}$ and $\mathrm{F}_{2}$ - generations, respectively. This result may be due to the Sids-1 $\left(\mathrm{P}_{7}\right)$ reveal to be the tallest parent and passed it target genes to this cross (Table 4). These results are in agreement with those obtained by Wajid et al. (2011), Nassar (2013), Hassan (2015), Hassan (2016), Jaiswal et al. (2017), Asmaa et al (2018), Sharma et al (2019) and Bajaniya et al (2019).

\section{0-grains weight $(\mathrm{g})$ :}

Regarding 100-grains weight, mean of the parents ranged from 3.49 and $3.15 \mathrm{~g}$ for Line $158\left(\mathrm{P}_{1}\right)$ to 5.87 and $4.66 \mathrm{~g}$ for Gemmiza-11 $\left(\mathrm{P}_{3}\right)$ with an average of 4.73 and $3.54 \mathrm{~g}$ at normal and late planting dates, respectively (Table 5).

With respect to the average of 100grain weight for $\mathrm{F}_{1}$-hybrids, it noticed that the heaviest $F_{1}$-hybrids were Shamiss- $3 \times$ Sids- 1 (6.41) and Line158× Gemmiza-11 (4.93), while the lightest were Misr-2 $\times$ Shandweel-1 (4.02) and Gemmiza-11 × Sakha-93 (2.93) under normal and late planting dates, respectively (Table 4).

The $\mathrm{F}_{2}$-population performance ranged from 3.93 for Gemmiza-11 $\times$ Sakha93 and 3.00 for Line $158 \times$ Giza-168 to 5.45 and 4.86 for Shamiss- $3 \times$ Gemmiza-11 with an average of 4.57 and $3.58 \mathrm{~g}$ under normal and late planting dates, respectively.

100-grain weight overall genotypes was decreased by heat stress, the decrease reached to 24.95 and $22.56 \%$ from normal date in $F_{1}$ 's and $F_{2}$-populations, respectively. This reduction may be due to the effects of the high temperature during grain filling period (Table 4). In this connection, Samra et al. (1989) showed that high temperature and strong dry winds during month of maturity may have a forced effect on maturity of late plant crop and result low weight. These findings are in line with those reported by Darwish et al (2006), Nassar (2013), Hassan (2015), Hassan (2016), Jaiswal et al (2017), Asmaa et al (2018), Sharma et al (2019) and Bajaniya et al (2019).

\section{Grain yield/plant (g):}

Concerning grain yield/plant, the average overall parents were 16.07 and 10.65 $\mathrm{g}$ under both planting dates, respectively. The parental genotypes Line158 $\left(\mathrm{P}_{1}\right)$ and $\mathrm{P}_{3}$ had the highest yield (19.46 g) and (12.08 g) under normal and late planting dates, respectively. While under both growing dates, the parent $\mathrm{P}_{6}$ gave the lowest values; 12.84 and 9.36, respectively (Table 5).

The average of grain yield/plant for $F_{1}$-hybrids showed that the high averages were $24.66 \mathrm{gm}$ for Shamiss-3 $\times$ Giza-168 and $16.14 \mathrm{~g}$ for Line158x Sids-1, while the low averages were $13.13 \mathrm{gm}$ for Gemmiza- $11 \times$ Giza-168 and 7.26 g for Sakha-93 $\times$ Misr-2 at both sowing dates, respectively. The average grain yield/plant for the $\mathrm{F}_{1}$-hybrids was 18.29 and $12.01 \mathrm{~g}$ under both planting dates, respectively.

With regard to grain yield/plant, the average $F_{2}$-populations showed that the high averages were 17.83 and $13.68 \mathrm{~g}$ for Shandweel- $1 \times$ Sids-1, while the low averages were $9.55 \mathrm{~g}$ for Line158 $\times$ Misr-2 and 6.26 gm for Sakha-93 $\times$ Misr-2 under normal and late planting dates, respectively. The average grain yield/plant for the $\mathrm{F}_{2}$-populations were 14.34 and $10.25 \mathrm{~g}$ under normal and late planting dates, respectively. Grain yield/plant overall genotypes was decreased by heat stress, the decrease reached to 34.20 and $29.88 \%$ from normal date in the $\mathrm{F}_{1}$ 's and $\mathrm{F}_{2}$ populations, respectively. This reduction may be because of high temperature on grain production rate. These results are in line with 
those obtained by Dencic et al (2000), Hoffman and Burucs (2005), Mohamed (2007), Nassar (2013), Hassan (2015), Hassan (2016), Jaiswal et al. (2017), Asmaa et al (2018), Sharma et al (2019) and Bajaniya et al (2019).

\section{Heat susceptibility index (HSI) and sensitivity (S):}

Heat susceptibility index and sensitivity were used to estimate relative stress injury where it accounted for variation in yield potential and stress intensity. Low both heat susceptibility index (HSI $<1)$ and sensitivity $(\mathrm{S}<1)$ are indication to high stress tolerance (Fischer and Maurer; 1978) and less sensitive to heat according to sensitivity test of Falconer (1990). Heat susceptibility index for grain yield/plant (Table 5), indicated that the most tolerant parents were Gemmiza 11 (P3), Sakha 93 (P4), Shandweel 1(P6) and Giza 168 (P8). In addition, the most tolerant of both $\mathrm{F}_{1}$-hybrids and $\mathrm{F}_{2}$-crosses were ; Line $158 \times$ Gemmiza 11 , Line $158 \times$ Shandweel 1 , Line $158 \times$ Giza 168 , Shamiss $3 \times$ Gemmiza 11, Shamiss $3 \times$ Misr 2, and Gemmiza 11× Giza 168 . These parents and both $F_{1}$-hybrids and $\mathrm{F}_{2}$ crosses could be considered moderately sensitive to heat according to sensitivity test of Falconer (1990). Similar results were obtained by Zakaria (1999), Taghian and Abo-Elwafa (2003), Nassar (2013) and Hassan (2015).

\section{Combining ability:}

The data in Table 3 presented that mean squares due to GCA and SCA were highly significant for all studied traits under both dates, except grain yield/plant for GCA in $F_{1}$ and $F_{2}$-generations under normal and late dates. These results indicated that differences due to additive and non-additive gene actions were influencing the most studied traits. GCA/SCA ratio was less than one for days to heading, 100-grain yield and grain yield/plant in the $F_{1}$ and $F_{2}$-generations under both environments, indicating that dominance genetic effects were predominant and played the major effect in the control mechanism of these traits. In the same time, plant height exhibited more additive effects. Similar results were reached in other studies (Nayeem and veer, 2000; Khieralla et al, 2001; Ashoush et al, 2001; Ahmed, 2003; Ali, 2006; El-Karamity et al, 2007; Ahmed and Mohamed; 2009and Hassan 2015).

\section{Days to heading date (day):}

The parents Gemmiza $11\left(\mathrm{P}_{3}\right)$ and Sakha $93 \quad\left(\mathrm{P}_{4}\right)$ showed highly significant negative GCA effects under both planting dates at $F_{1}$ and $F_{2}$-generations (Table 6). This indicates that these two parents were good combiners for days to heading date. Thus, these parents may possess favorable genes which could be utilized in breeding programs to improve the earliness trait in wheat. On the other hand, the two parents, Shamiss $3\left(\mathrm{P}_{2}\right)$ and Misr $2\left(\mathrm{P}_{5}\right)$, showed highly significant positive GCA effects at normal and late planting dates in both generations.

Estimates of SCA effects, Table (5) for $F_{1}$ and $F_{2}$ generations showed that six crosses ; Shamiss-3 $\times$ Gemmiza-11, Shamiss$3 \times$ Sids-1, Shamiss- $3 \times$ Giza-168, Gemmiza$11 \times$ Misr-2, Sakha-93 $\times$ Sids-1 and Misr-2 $\times$ Sids-1 showed negative and significant or highly significant SCA effects for both generations at both planting dates. Thus, these crosses could be considering the best combinations in both generations for days to heading date, so they might be exploited for breeding earlier varieties. These results are in agreement with those reported by Akinci (2009), Nassar (2013), Hassan (2015), Hassan (2016), Jaiswal et al (2017), Asmaa et al 
(2018), Sharma et al (2019) and Bajaniya et al (2019).

\section{Plant height $(\mathbf{c m})$ :}

Concerning plant height, three parents; Gemmiza-11 $\left(\mathrm{P}_{3}\right)$, Misr-2 $\left(\mathrm{P}_{5}\right)$ and Giza-168 $\left(\mathrm{P}_{8}\right)$ had highly significant and positive GCA effects in $F_{1}$ and $F_{2}$-generations under both planting dates (Table 6). This indicates that these parents could be the good combiners for plant height at both generations and different dates. On the other hand, the parents Line158 $\left(\mathrm{P}_{1}\right)$, Shamiss-3 $\left(\mathrm{P}_{2}\right)$ and Sakha-93 $\left(\mathrm{P}_{4}\right)$ showed negative and highly significant GCA effects in $F_{1}$ and $F_{2}$ under both sowing dates.

With respect to SCA for plant height, the cross Shamiss-3 $\times$ Giza-168 gave positive and highly significant SCA effects of both generations under both sowing dates. Also, the hybrid Misr-2 $\times$ Shandweel-1 showed the same trend under late planting date. Few number of either $F_{1}$ 's or $F_{2}$-populations exhibited SCA negative effects under both environments (Table 6). Most of these hybrids were shorter than over all means, revealing that these crosses could be considered for breeding program for developing semi-dwarf under area conditions. Similar results were obtained by Ahmed, 2003, Ali, 2006, El-Karamity et al, 2007, Hassan 2015, Jaiswal et al (2017), Asmaa et al (2018), Sharma et al (2019) and Bajaniya et al (2019).

\section{0-grain weight (gm):}

With respect to 100-grain weight, the parents Shamiss-3 $\left(\mathrm{P}_{2}\right)$ and Gemmiza-11 $\left(\mathrm{P}_{3}\right)$ were positive and highly significant GCA of $F_{1, s}$ and $F_{2}$-populations at both environments (Table 7). Therefore, it could be considered good combiner for 100-grain weight.

Three crosses; Line158 $\times$ Gemmiza-11, Shamiss-3 $\times$ Sakha-93 and Shamiss-3× Giza-
168 were highly significant and positive SCA of $F_{1, s}$ and $F_{2}$-populations under both planting dates (Table 7). It is noticed that, these crosses involved low $\times$ high and high $\times$ low general combiner parents for this trait. Similar results were obtained by Nagwa (2007), ), Nassar (2013), Hassan (2015), Hassan (2016), Jaiswal et al. (2017), Asmaa et al (2018), Sharma et al (2019) and Bajaniya et al (2019).

\section{Grain yield/plant (g):}

Concerning grain yield/plant, only one parent $\left(\mathrm{P}_{2}\right)$ had highly significant and positive GCA of both generations under two planting dates (Table 7). This parent could be seen as a good combiner for grain yield/plant. The two crosses Shamiss-3 × Giza-168 and Sakha-93× Giza-168 gave highly significant and positive SCA effects of $F_{1}$ and $F_{2}$-generations at both planting dates (Table 7). These two crosses included high $\times$ low and low $\times$ low general combiner parents. These results were agree with those concluded by Nayeem and Veer (2000), Ahmed and Mohamed (2009), Akinci (2009), Nassar (2013), Hassan (2015), Hassan (2016), Jaiswal et al (2017), Asmaa et al (2018), Sharma et al (2019) and Bajaniya et al (2019). From previous, it is reveal that the parent Gemmiza-11 $\left(\mathrm{P}_{3}\right)$ was considered as the best combiner for heading date, plant height and 100-grain weight, but the parent Shamiss-3 $\left(\mathrm{P}_{2}\right)$ was considered as the best combiner of $\mathrm{F}_{1, \mathrm{~s}}$ and $\mathrm{F}_{2}$-generations for 100grain weight and grain yield/plant under both dates. The cross Shamiss- $3 \times$ Giza-168 could be considered as the best combination in $F_{1}$ and $F_{2}$ generations for the studied characters at both growing dates. 
Table 1. Some physical and chemical properties of the experimental site.

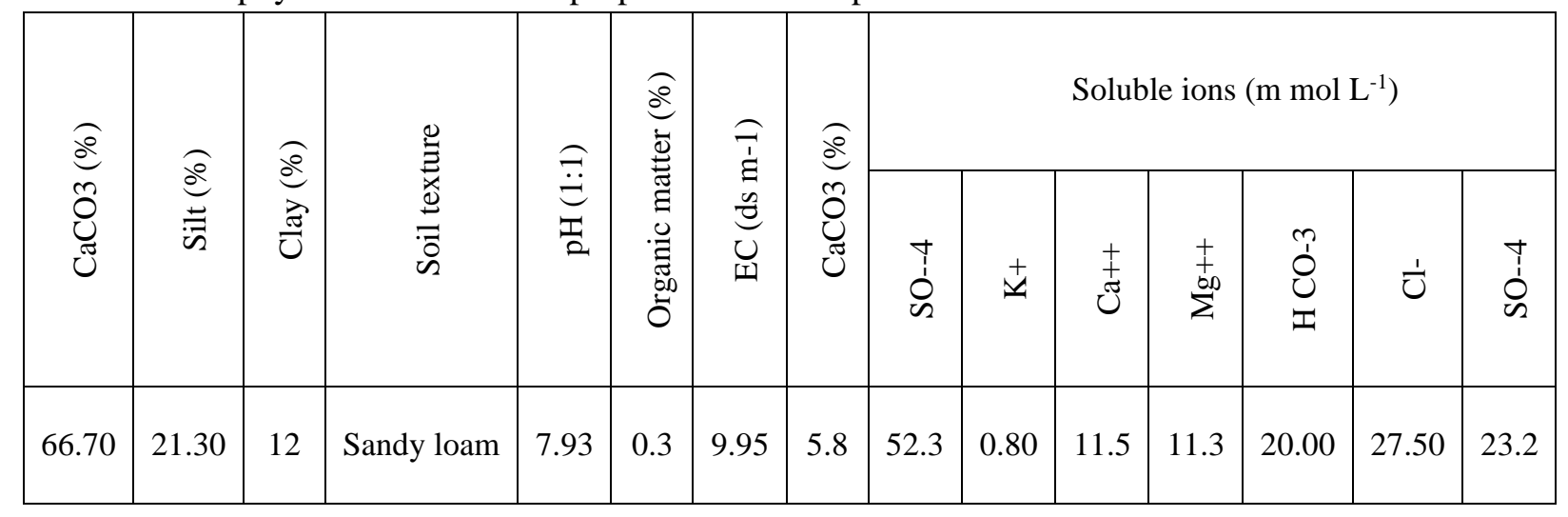

Table 2. Maximum, minimum and average monthly temperature $\left({ }^{\circ} \mathrm{C}\right)$ at Qena from heading date to physiological maturity stage during 2017/2018 season.

\begin{tabular}{|c|c|c|c|c|}
\hline \multicolumn{2}{|c|}{ Season } & \multicolumn{3}{|c|}{$2017 / 2018$} \\
\hline \multirow[t]{2}{*}{ Month } & Day & Maximum & Minimum & Average \\
\hline & $22-30$ & 21.61 & 17.26 & 19.39 \\
\hline \multicolumn{2}{|c|}{ Average } & 21.61 & 17.26 & 19.39 \\
\hline \multirow{3}{*}{ December } & $1-10$ & 18.82 & 15.68 & 16.70 \\
\hline & $11-20$ & 16.27 & 12.59 & 14.37 \\
\hline & $21-31$ & 18.34 & 12.35 & 13.98 \\
\hline \multicolumn{2}{|c|}{ Average } & 17.81 & 13.54 & 15.02 \\
\hline \multirow{3}{*}{ January } & $1-10$ & 16.39 & 13.03 & 14.81 \\
\hline & $11-20$ & 17.12 & 13.19 & 15.04 \\
\hline & $21-31$ & 15.37 & 11.95 & 14.03 \\
\hline \multicolumn{2}{|c|}{ Average } & 16.29 & 12.72 & 14.63 \\
\hline \multirow{3}{*}{ February } & $1-10$ & 24.78 & 14.71 & 20.22 \\
\hline & $11-20$ & 24.51 & 14.91 & 18.09 \\
\hline & $21-28$ & 25.25 & 18.98 & 21.71 \\
\hline \multicolumn{2}{|c|}{ Average } & 24.85 & 16.20 & 20.01 \\
\hline \multirow{3}{*}{ March } & $1-10$ & 27.03 & 21.3 & 24.17 \\
\hline & $11-20$ & 28.58 & 21.28 & 24.51 \\
\hline & $21-31$ & 31.06 & 22.1 & 26.15 \\
\hline \multicolumn{2}{|c|}{ Average } & 28.89 & 21.56 & 24.94 \\
\hline \multirow{3}{*}{ April } & $1-10$ & 34.43 & 25.05 & 28.08 \\
\hline & $11-20$ & 30.67 & 23.31 & 27.00 \\
\hline & $21-30$ & 32.83 & 27.54 & 30.73 \\
\hline \multicolumn{2}{|c|}{ Average } & 32.64 & 25.30 & 28.60 \\
\hline May & $1-15$ & 35.70 & 28.48 & 31.59 \\
\hline \multicolumn{2}{|c|}{ Average } & 35.70 & 28.48 & 31.59 \\
\hline
\end{tabular}

Source: Meteorological Authority, Qena station, at South Valley University. 
Table 3. Mean squares from ANOVA combining ability analysis in the $F_{1}$-hybrids and $F_{2}-$ populations of bread wheat crosses under normal $(\mathrm{N})$ and late $(\mathrm{L})$ planting dates during 2017/2018 season.

\begin{tabular}{|c|c|c|c|c|c|c|c|c|c|}
\hline \multirow{3}{*}{ S.O.V } & \multirow{3}{*}{ df } & \multicolumn{4}{|c|}{ Days to heading (days) } & \multicolumn{4}{|c|}{ Plant height $(\mathrm{cm})$} \\
\hline & & \multicolumn{2}{|c|}{$\mathrm{F}_{1}$} & \multicolumn{2}{|c|}{$\mathrm{F}_{2}$} & \multicolumn{2}{|c|}{$\mathrm{F}_{1}$} & \multicolumn{2}{|c|}{$\mathrm{F}_{2}$} \\
\hline & & $\mathrm{N}$ & $\mathrm{L}$ & $\mathrm{N}$ & $\mathrm{L}$ & $\mathrm{N}$ & $\mathrm{L}$ & $\mathrm{N}$ & $\mathrm{L}$ \\
\hline Reps & 2 & $38.62^{* *}$ & $4.34^{*}$ & $4.15^{*}$ & 3.45 & $47.90^{* *}$ & $12.62^{*}$ & $195.26^{* *}$ & $71.52^{* *}$ \\
\hline Genotypes(G) & 35 & $21.51^{* *}$ & $23.31^{* * *}$ & $61.84^{* *}$ & $26.18^{* *}$ & $79.31^{* *}$ & $38.65^{* *}$ & $88.07^{* *}$ & $69.10^{* * *}$ \\
\hline Parents $(\mathrm{P})$ & 7 & $26.57^{* *}$ & $52.17^{* *}$ & $26.57^{* *}$ & $52.17^{* *}$ & $138.99^{* *}$ & $64.72^{* *}$ & $138.99^{* *}$ & $64.72^{* *}$ \\
\hline Crosses (C) & 27 & $8.64^{* * *}$ & $4.49^{* * *}$ & $4.31^{* *}$ & $4.18^{* * *}$ & $54.75^{* *}$ & $30.93^{* *}$ & $61.82^{* *}$ & $71.53^{* *}$ \\
\hline $\mathrm{P}$ vs $\mathrm{C}$ & 1 & $333.40^{* *}$ & $329.65^{* * *}$ & $1862.22^{* *}$ & $438.22^{* *}$ & $324.58^{* *}$ & $64.77^{* *}$ & $440.37^{* *}$ & $34.13^{* *}$ \\
\hline GCA & 7 & $11.14^{* *}$ & $12.47^{* * *}$ & $6.43^{* *}$ & $11.28^{* *}$ & $93.69^{* *}$ & $47.89^{* *}$ & $109.67^{* *}$ & $52.42^{* * *}$ \\
\hline SCA & 28 & $6.18^{* *}$ & $6.60^{* *}$ & $24.16^{* * *}$ & $8.09^{* * *}$ & $9.62^{* *}$ & $4.13^{* *}$ & 9.28 & $15.69^{* * *}$ \\
\hline Error & 70 & 1.61 & 1.22 & 1.25 & 1.17 & 8.27 & 3.50 & 8.85 & 4.66 \\
\hline$\sum \mathrm{g}_{\mathrm{i}}{ }^{2} / \Sigma \mathrm{S}_{\mathrm{ij}}{ }^{2}$ & - & 0.19 & 0.19 & 0.03 & 0.14 & 1.32 & 1.58 & 1.69 & 0.36 \\
\hline \multirow{3}{*}{ S.O.V } & \multirow{3}{*}{$\mathrm{df}$} & \multicolumn{4}{|c|}{ 100-grain weight (g) } & \multicolumn{4}{|c|}{ Grain yield/plant $(\mathrm{g})$} \\
\hline & & \multicolumn{2}{|c|}{$\overline{F_{1}}$} & \multicolumn{2}{|c|}{$\overline{F_{2}}$} & \multicolumn{2}{|c|}{$F_{1}$} & \multicolumn{2}{|c|}{$\mathrm{F}_{2}$} \\
\hline & & $\mathrm{N}$ & $\mathrm{L}$ & $\mathrm{N}$ & $\mathrm{L}$ & $\mathrm{N}$ & $\mathrm{L}$ & $\mathrm{N}$ & $\mathrm{L}$ \\
\hline Reps & 2 & 0.23 & $0.38^{* *}$ & $0.41^{*}$ & $0.20^{* * *}$ & $47.66^{* *}$ & 4.14 & $29.14^{* *}$ & $7.33^{* *}$ \\
\hline Genotypes(G) & 35 & $1.15^{* *}$ & $0.49^{* *}$ & $0.73^{* *}$ & $1.03^{* *}$ & $42.31^{* *}$ & $10.20^{* * *}$ & $16.55^{* *}$ & $9.31^{* *}$ \\
\hline Parents (P) & 7 & $1.28^{* *}$ & $0.68^{* *}$ & $1.28^{* *}$ & $0.68^{* *}$ & $29.94^{*}$ & $8.37^{* *}$ & $29.94^{* *}$ & $8.37^{* *}$ \\
\hline Crosses (C) & 27 & $1.14^{* *}$ & $0.45^{* *}$ & $0.59^{* *}$ & $1.15^{* *}$ & $42.32^{* *}$ & $10.43^{* *}$ & $12.48^{* *}$ & $9.46^{* *}$ \\
\hline $\mathrm{P}$ vs $\mathrm{C}$ & 1 & $0.52^{*}$ & $0.44^{* *}$ & $0.63^{*}$ & $0.25^{*}$ & $128.90^{* *}$ & $16.95^{* *}$ & $32.57^{* *}$ & $12.00^{* *}$ \\
\hline GCA & 7 & $0.43^{* *}$ & 0.15 & $0.45^{* *}$ & $0.68^{* * *}$ & 12.02 & 3.63 & 1.99 & 4.65 \\
\hline SCA & 28 & $0.37^{* *}$ & $0.17^{* *}$ & $0.19^{*}$ & $0.26^{* *}$ & $14.62^{*}$ & 3.34 & $6.40^{* * *}$ & $2.72^{*}$ \\
\hline Error & 70 & 0.13 & 0.07 & 0.11 & 0.04 & 8.06 & 2.59 & 1.69 & 1.55 \\
\hline$\Sigma \mathrm{g}_{\mathrm{i}}^{2} / \Sigma \mathrm{S}_{\mathrm{ij}}^{2}$ & - & 0.12 & 0.08 & 0.27 & 0.27 & 0.08 & 0.11 & 0.02 & 0.19 \\
\hline
\end{tabular}

$*$ and $* *$ : significant at 0.05 and 0.01 levels of probability, respectively. 
Table 4. Mean performance of the eight parents, $F_{1}$ 's hybrids and $F_{2}$ 's populations for days to heading, plant height and 100-grain weight under normal $(\mathrm{N})$ and late (L) planting dates in 2017/2018 season.

\begin{tabular}{|c|c|c|c|c|c|c|c|c|c|c|c|c|}
\hline \multirow[t]{3}{*}{ Genotypes } & \multicolumn{4}{|c|}{ Days to heading (days) } & \multicolumn{4}{|c|}{ Plant height $(\mathrm{cm})$} & \multicolumn{4}{|c|}{100 -grain weight (g) } \\
\hline & \multicolumn{2}{|c|}{$\mathrm{F}_{1}$} & \multicolumn{2}{|c|}{$\mathrm{F}_{2}$} & \multicolumn{2}{|c|}{$\mathrm{F}_{1}$} & \multicolumn{2}{|c|}{$\mathrm{F}_{2}$} & \multicolumn{2}{|c|}{$\mathrm{F}_{1}$} & \multicolumn{2}{|c|}{$\mathrm{F}_{2}$} \\
\hline & $\mathrm{N}$ & $\mathrm{L}$ & $\mathrm{N}$ & $\mathrm{L}$ & $\mathrm{N}$ & $\mathrm{L}$ & $\mathrm{N}$ & $\mathrm{L}$ & $\mathrm{N}$ & $\mathrm{L}$ & $\mathrm{N}$ & $\mathrm{L}$ \\
\hline $\mathrm{P}_{1}$ & 87.00 & 78.33 & 87.00 & 78.33 & 66.49 & 53.41 & 66.49 & 53.41 & 3.49 & 3.15 & 3.49 & 3.15 \\
\hline $\mathrm{P}_{2}$ & 89.33 & 78.00 & 89.33 & 78.00 & 56.91 & 53.73 & 56.91 & 53.73 & 4.78 & 3.22 & 4.78 & 3.22 \\
\hline $\mathrm{P}_{3}$ & 83.00 & 70.33 & 83.00 & 70.33 & 69.11 & 56.45 & 69.11 & 56.45 & 5.87 & 4.66 & 5.87 & 4.66 \\
\hline $\mathrm{P}_{4}$ & 81.33 & 70.67 & 81.33 & 70.67 & 57.11 & 47.96 & 57.11 & 47.96 & 4.59 & 3.44 & 4.59 & 3.44 \\
\hline $\mathrm{P}_{5}$ & 88.67 & 78.33 & 88.67 & 78.33 & 73.40 & 58.24 & 73.40 & 58.24 & 4.76 & 3.39 & 4.76 & 3.39 \\
\hline $\mathrm{P}_{6}$ & 84.00 & 71.33 & 84.00 & 71.33 & 65.26 & 55.92 & 65.26 & 55.92 & 4.72 & 3.42 & 4.72 & 3.42 \\
\hline $\mathrm{P}_{7}$ & 86.67 & 81.00 & 86.67 & 81.00 & 75.64 & 63.11 & 75.64 & 63.11 & 5.06 & 3.39 & 5.06 & 3.39 \\
\hline $\mathrm{P}_{8}$ & 82.67 & 74.00 & 82.67 & 74.00 & 68.65 & 60.36 & 68.65 & 60.36 & 4.57 & 3.62 & 4.57 & 3.62 \\
\hline Mean $(p)$ & 85.33 & 75.25 & 85.33 & 75.25 & 66.57 & 56.15 & 66.57 & 56.15 & 4.73 & 3.54 & 4.73 & 3.54 \\
\hline $\mathrm{P}_{1} \times \mathrm{P}_{2}$ & 82.00 & 70.00 & 75.67 & 70.67 & 65.13 & 51.53 & 66.49 & 53.11 & 4.42 & 3.30 & 4.22 & 3.30 \\
\hline $\mathrm{P}_{1} \times \mathrm{P}_{3}$ & 80.00 & 69.33 & 74.33 & 68.67 & 68.49 & 56.49 & 72.38 & 66.21 & 5.53 & 4.93 & 5.25 & 4.57 \\
\hline $\mathrm{P}_{1} \times \mathrm{P}_{4}$ & 81.00 & 69.33 & 75.00 & 69.33 & 63.13 & 48.66 & 62.44 & 50.12 & 5.23 & 3.77 & 4.30 & 3.29 \\
\hline $\mathrm{P}_{1} \times \mathrm{P}_{5}$ & 82.00 & 70.67 & 80.00 & 69.33 & 70.91 & 55.88 & 73.38 & 65.60 & 5.25 & 3.91 & 4.62 & 3.19 \\
\hline $\mathrm{P}_{1} \times \mathrm{P}_{6}$ & 78.33 & 72.00 & 73.00 & 68.67 & 66.22 & 52.73 & 71.18 & 55.35 & 5.06 & 3.83 & 4.39 & 3.31 \\
\hline $\mathrm{P}_{1} \times \mathrm{P}_{7}$ & 82.33 & 72.00 & 75.00 & 70.00 & 68.13 & 51.17 & 71.75 & 52.49 & 5.04 & 3.72 & 4.64 & 3.46 \\
\hline $\mathrm{P}_{1} \times \mathrm{P}_{8}$ & 80.33 & 70.00 & 74.00 & 69.33 & 65.20 & 54.53 & 71.20 & 57.03 & 4.84 & 3.77 & 4.37 & 3.00 \\
\hline $\mathrm{P}_{2} \times \mathrm{P}_{3}$ & 81.00 & 68.67 & 75.00 & 68.67 & 69.62 & 55.05 & 70.02 & 55.36 & 5.06 & 3.78 & 5.45 & 4.86 \\
\hline $\mathrm{P}_{2} \times \mathrm{P}_{4}$ & 82.00 & 70.67 & 76.00 & 69.33 & 66.07 & 49.98 & 60.71 & 49.53 & 6.13 & 3.80 & 5.27 & 4.58 \\
\hline $\mathrm{P}_{2} \times \mathrm{P}_{5}$ & 85.00 & 72.00 & 75.67 & 71.33 & 72.56 & 55.78 & 68.96 & 55.30 & 5.69 & 3.87 & 4.96 & 3.21 \\
\hline $\mathrm{P}_{2} \times \mathrm{P}_{6}$ & 83.00 & 72.00 & 74.00 & 70.00 & 68.82 & 52.09 & 69.80 & 52.50 & 4.86 & 3.85 & 5.03 & 3.48 \\
\hline $\mathrm{P}_{2} \times \mathrm{P}_{7}$ & 82.33 & 72.00 & 75.00 & 70.00 & 71.31 & 54.22 & 69.60 & 52.91 & 6.41 & 4.30 & 4.65 & 3.67 \\
\hline $\mathrm{P}_{2} \times \mathrm{P}_{8}$ & 81.33 & 70.67 & 76.00 & 70.67 & 74.18 & 52.63 & 73.04 & 64.91 & 5.83 & 4.06 & 5.16 & 4.51 \\
\hline $\mathrm{P}_{3} \times \mathrm{P}_{4}$ & 82.00 & 68.67 & 74.33 & 69.33 & 68.22 & 53.82 & 71.84 & 65.72 & 4.49 & 2.93 & 3.93 & 3.82 \\
\hline $\mathrm{P}_{3} \times \mathrm{P}_{5}$ & 81.00 & 70.00 & 75.33 & 70.00 & 74.16 & 59.33 & 74.29 & 65.63 & 4.87 & 3.85 & 4.52 & 3.25 \\
\hline $\mathrm{P}_{3} \times \mathrm{P}_{6}$ & 79.67 & 72.00 & 75.00 & 69.33 & 72.73 & 57.78 & 70.67 & 55.87 & 4.61 & 3.47 & 4.48 & 3.17 \\
\hline $\mathrm{P}_{3} \times \mathrm{P}_{7}$ & 81.67 & 72.00 & 76.67 & 71.33 & 80.78 & 58.32 & 76.06 & 57.60 & 4.91 & 3.69 & 5.40 & 4.62 \\
\hline $\mathrm{P}_{3} \times \mathrm{P}_{8}$ & 78.00 & 68.67 & 75.00 & 70.00 & 74.40 & 58.53 & 71.98 & 57.83 & 4.09 & 3.42 & 4.16 & 3.40 \\
\hline $\mathrm{P}_{4} \times \mathrm{P}_{5}$ & 82.33 & 72.00 & 75.67 & 72.00 & 69.71 & 53.80 & 69.85 & 57.13 & 4.31 & 3.37 & 3.98 & 3.37 \\
\hline $\mathrm{P}_{4} \times \mathrm{P}_{6}$ & 81.00 & 72.00 & 74.67 & 71.33 & 67.60 & 49.24 & 66.07 & 53.41 & 4.44 & 4.01 & 4.03 & 3.31 \\
\hline $\mathrm{P}_{4} \times \mathrm{P}_{7}$ & 77.67 & 71.33 & 75.00 & 70.00 & 70.11 & 52.20 & 70.47 & 53.17 & 4.58 & 3.70 & 4.63 & 3.22 \\
\hline $\mathrm{P}_{4} \times \mathrm{P}_{8}$ & 77.67 & 71.33 & 75.00 & 70.00 & 65.73 & 48.64 & 65.31 & 54.96 & 4.59 & 3.55 & 4.11 & 3.25 \\
\hline $\mathrm{P}_{5} \times \mathrm{P}_{6}$ & 81.67 & 72.00 & 76.00 & 72.00 & 72.91 & 59.53 & 74.82 & 60.42 & 4.02 & 3.03 & 4.02 & 3.36 \\
\hline $\mathrm{P}_{5} \times \mathrm{P}_{7}$ & 79.67 & 72.00 & 75.00 & 72.00 & 77.47 & 58.45 & 78.60 & 60.26 & 4.35 & 3.38 & 5.02 & 3.36 \\
\hline $\mathrm{P}_{5} \times \mathrm{P}_{8}$ & 81.67 & 72.00 & 76.00 & 72.00 & 77.69 & 57.49 & 77.11 & 62.49 & 5.48 & 3.41 & 4.82 & 3.95 \\
\hline $\mathrm{P}_{6} \times \mathrm{P}_{7}$ & 82.33 & 72.00 & 75.67 & 72.00 & 75.29 & 53.56 & 77.27 & 57.37 & 4.26 & 3.41 & 4.26 & 3.41 \\
\hline $\mathrm{P}_{6} \times \mathrm{P}_{8}$ & 82.33 & 72.00 & 75.67 & 72.00 & 70.84 & 53.64 & 74.67 & 59.67 & 4.54 & 3.64 & 4.12 & 3.12 \\
\hline $\mathrm{P}_{7} \times \mathrm{P}_{8}$ & 81.67 & 72.00 & 76.00 & 72.00 & 73.36 & 54.93 & 80.07 & 58.08 & 4.22 & 3.61 & 4.55 & 4.22 \\
\hline Mean (C) & 81.11 & 71.05 & 75.35 & 70.40 & 70.74 & 54.29 & 71.43 & 57.50 & 4.90 & 3.69 & 4.57 & 3.58 \\
\hline Mean $(G)$ & 83.22 & 73.15 & 80.34 & 72.33 & 68.66 & 55.22 & 69.00 & 56.82 & 4.81 & 3.61 & 4.61 & 3.57 \\
\hline LSD'0.05 P) & 2.21 & 1.86 & 1.88 & 1.82 & 5.05 & 3.17 & 5.22 & 3.78 & 0.65 & 0.47 & 0.56 & 0.37 \\
\hline LSD'0.05(C) & 2.17 & 1.90 & 2.06 & 1.86 & 4.65 & 2.91 & 4.81 & 3.34 & 0.57 & 0.43 & 0.51 & 0.31 \\
\hline LSD'0.05 G) & 1.94 & 1.63 & 1.61 & 1.59 & 4.42 & 2.88 & 4.57 & 3.31 & 0.56 & 0.44 & 0.51 & 0.31 \\
\hline Red.\% & \multicolumn{2}{|c|}{12.10} & \multicolumn{2}{|c|}{9.97} & \multicolumn{2}{|c|}{19.58} & \multicolumn{2}{|c|}{17.65} & & & \multicolumn{2}{|c|}{22.56} \\
\hline
\end{tabular}


Table 5. Mean performance, heat susceptibility index (HSI) and sensitivity (S) for grain yield/plant of parents, $F_{1}$-hybrids and $F_{2}$-populations under normal $(\mathrm{N})$ and late $(\mathrm{L})$ planting dates during 2017/2018 season.

\begin{tabular}{|c|c|c|c|c|c|c|c|c|}
\hline \multirow[t]{3}{*}{ Genotypes } & \multicolumn{4}{|c|}{ Grain yield/plant (gm) } & \multirow{2}{*}{\multicolumn{2}{|c|}{ HSI }} & \multirow{2}{*}{\multicolumn{2}{|c|}{$\mathrm{S}$}} \\
\hline & \multicolumn{2}{|c|}{$\mathrm{F}_{1}$} & \multicolumn{2}{|c|}{$\mathrm{F}_{2}$} & & & & \\
\hline & $\mathrm{N}$ & $\mathrm{L}$ & $\mathrm{N}$ & $\mathrm{L}$ & $\mathrm{F}_{1}$ & $\mathrm{~F}_{2}$ & $\mathrm{~F}_{1}$ & $\mathrm{~F}_{2}$ \\
\hline $\mathrm{P}_{1}$ & 19.46 & 9.78 & 19.46 & 9.78 & \multirow{2}{*}{\multicolumn{2}{|c|}{$\begin{array}{l}1.72 \\
1.43\end{array}$}} & \multicolumn{2}{|c|}{1.85} \\
\hline $\mathrm{P}_{2}$ & 17.91 & 10.46 & 17.91 & 10.46 & & & \multicolumn{2}{|c|}{1.42} \\
\hline $\mathrm{P}_{3}$ & 13.59 & 12.08 & 13.59 & 12.08 & \multicolumn{2}{|c|}{0.38} & \multicolumn{2}{|c|}{0.29} \\
\hline $\mathrm{P}_{4}$ & 13.70 & 10.29 & 13.70 & 10.29 & \multicolumn{2}{|c|}{0.86} & \multicolumn{2}{|c|}{0.65} \\
\hline $\mathrm{P}_{5}$ & 19.13 & 11.70 & 19.13 & 11.70 & \multicolumn{2}{|c|}{1.34} & \multicolumn{2}{|c|}{1.42} \\
\hline $\mathrm{P}_{6}$ & 12.84 & 9.36 & 12.84 & 9.36 & \multicolumn{2}{|c|}{0.93} & \multicolumn{2}{|c|}{0.66} \\
\hline $\mathrm{P}_{7}$ & 17.38 & 10.25 & 17.38 & 10.25 & \multicolumn{2}{|c|}{1.41} & \multirow{2}{*}{\multicolumn{2}{|c|}{$\begin{array}{l}1.36 \\
0.62\end{array}$}} \\
\hline $\mathrm{P}_{8}$ & 14.51 & 11.27 & 14.51 & 11.27 & \multicolumn{2}{|c|}{0.77} & & \\
\hline Mean $p$ & 16.07 & 10.65 & 16.07 & 10.65 & - & - & - & - \\
\hline $\mathrm{P}_{1} \times \mathrm{P}_{2}$ & 18.18 & 11.80 & 15.36 & 10.80 & 1.03 & 1.02 & 1.02 & 1.11 \\
\hline $\mathrm{P}_{1} \times \mathrm{P}_{3}$ & 13.72 & 11.90 & 12.68 & 9.85 & 0.39 & 0.77 & 0.29 & 0.69 \\
\hline $\mathrm{P}_{1} \times \mathrm{P}_{4}$ & 19.14 & 11.00 & 14.26 & 7.77 & 1.25 & 1.57 & 1.30 & 1.59 \\
\hline $\mathrm{P}_{1} \times \mathrm{P}_{5}$ & 20.27 & 13.50 & 9.55 & 9.53 & 0.98 & 0.01 & 1.08 & 0.00 \\
\hline $\mathrm{P}_{1} \times \mathrm{P}_{6}$ & 18.13 & 12.28 & 13.63 & 9.89 & 0.95 & 0.95 & 0.93 & 0.91 \\
\hline $\mathrm{P}_{1} \times \mathrm{P}_{7}$ & 18.34 & 12.14 & 16.14 & 8.48 & 0.99 & 1.64 & 0.99 & 1.87 \\
\hline $\mathrm{P} 1 \times \mathrm{P}_{8}$ & 15.30 & 11.06 & 15.29 & 11.43 & 0.82 & 0.87 & 0.68 & 0.94 \\
\hline $\mathrm{P}_{2} \times \mathrm{P}_{3}$ & 14.52 & 13.15 & 13.69 & 12.15 & 0.28 & 0.39 & 0.22 & 0.38 \\
\hline $\mathrm{P}_{2} \times \mathrm{P}_{4}$ & 14.01 & 13.32 & 14.40 & 10.07 & 0.14 & 1.04 & 0.11 & 1.06 \\
\hline $\mathrm{P}_{2} \times \mathrm{P}_{5}$ & 14.64 & 10.88 & 15.36 & 12.67 & 0.76 & 0.60 & 0.60 & 0.66 \\
\hline $\mathrm{P}_{2} \times \mathrm{P}_{6}$ & 18.21 & 10.73 & 12.56 & 10.62 & 1.21 & 0.53 & 1.19 & 0.47 \\
\hline $\mathrm{P}_{2} \times \mathrm{P}_{7}$ & 24.46 & 10.61 & 12.65 & 11.35 & 1.67 & 0.35 & 2.21 & 0.32 \\
\hline $\mathrm{P}_{2} \times \mathrm{P}_{8}$ & 24.66 & 12.80 & 14.66 & 11.92 & 1.41 & 0.64 & 1.89 & 0.67 \\
\hline $\mathrm{P}_{3} \times \mathrm{P}_{4}$ & 14.70 & 9.94 & 13.38 & 8.94 & 0.95 & 1.14 & 0.76 & 1.09 \\
\hline $\mathrm{P}_{3} \times \mathrm{P}_{5}$ & 19.94 & 12.36 & 16.29 & 11.10 & 1.12 & 1.10 & 1.21 & 1.27 \\
\hline $\mathrm{P}_{3} \times \mathrm{P}_{6}$ & 17.51 & 10.61 & 14.44 & 11.09 & 1.16 & 0.80 & 1.10 & 0.82 \\
\hline $\mathrm{P}_{3} \times \mathrm{P}_{7}$ & 16.28 & 11.93 & 15.21 & 8.72 & 0.79 & 1.47 & 0.69 & 1.59 \\
\hline $\mathrm{P}_{3} \times \mathrm{P}_{8}$ & 13.13 & 11.90 & 12.16 & 9.30 & 0.28 & 0.81 & 0.20 & 0.70 \\
\hline $\mathrm{P}_{4} \times \mathrm{P}_{5}$ & 14.11 & 7.26 & 13.50 & 6.26 & 1.43 & 1.85 & 1.09 & 1.77 \\
\hline $\mathrm{P}_{4} \times \mathrm{P}_{6}$ & 19.56 & 12.34 & 16.90 & 10.18 & 1.09 & 1.37 & 1.15 & 1.64 \\
\hline $\mathrm{P}_{4} \times \mathrm{P}_{7}$ & 24.28 & 11.43 & 10.33 & 9.85 & 1.56 & 0.16 & 2.05 & 0.12 \\
\hline $\mathrm{P}_{4} \times \mathrm{P}_{8}$ & 24.58 & 12.51 & 16.29 & 12.87 & 1.44 & 0.72 & 1.92 & 0.84 \\
\hline $\mathrm{P}_{5} \times \mathrm{P}_{6}$ & 15.61 & 8.64 & 12.37 & 7.64 & 1.31 & 1.32 & 1.11 & 1.16 \\
\hline $\mathrm{P}_{5} \times \mathrm{P}_{7}$ & 20.06 & 16.12 & 13.55 & 8.88 & 0.58 & 1.19 & 0.63 & 1.14 \\
\hline $\mathrm{P}_{5} \times \mathrm{P}_{8}$ & 18.62 & 12.31 & 14.18 & 11.73 & 1.00 & 0.60 & 1.00 & 0.60 \\
\hline $\mathrm{P}_{6} \times \mathrm{P}_{7}$ & 24.11 & 14.68 & 17.83 & 13.68 & 1.15 & 0.80 & 1.50 & 1.01 \\
\hline $\mathrm{P}_{6} \times \mathrm{P}_{8}$ & 22.24 & 15.85 & 17.49 & 8.16 & 0.85 & 1.84 & 1.02 & 2.28 \\
\hline $\mathrm{P}_{7} \times \mathrm{P}_{8}$ & 13.73 & 13.10 & 17.36 & 12.10 & 0.13 & 1.04 & 0.10 & 1.29 \\
\hline Mean C & 18.29 & 12.01 & 14.34 & 10.25 & - & - & - & - \\
\hline Mean (G) & 17.75 & 11.68 & 14.76 & 10.35 & - & - & - & - \\
\hline LSD' $(\mathrm{P})$ & 7.84 & 3.25 & 2.19 & 2.17 & - & - & - & - \\
\hline LSD' (C) & 6.57 & 2.79 & 2.10 & 1.92 & - & - & - & - \\
\hline LSD' $^{\prime}(\mathrm{G})$ & 6.51 & 2.76 & 1.99 & 1.98 & - & - & - & - \\
\hline Red. $\%$ & & & & & - & - & - & - \\
\hline
\end{tabular}


Table 6. General (GCA) and specific (SCA) combining ability effects for days to $50 \%$ heading and plant height of the parents and their $\mathrm{F}_{1}$-hybrids and $\mathrm{F}_{2}$-populations under normal $(\mathrm{N})$ and late (L) planting dates in 2017/2018 season.

\begin{tabular}{|c|c|c|c|c|c|c|c|c|}
\hline \multirow[t]{3}{*}{ Genotypes } & \multicolumn{4}{|c|}{ Days to heading date (day) } & \multicolumn{4}{|c|}{ Plant height $(\mathrm{cm})$} \\
\hline & \multicolumn{2}{|c|}{$\mathrm{F}_{2}$} & \multicolumn{2}{|c|}{$\mathrm{F}_{1}$} & \multicolumn{2}{|c|}{$\mathrm{F}_{2}$} & \multicolumn{2}{|c|}{$\mathrm{F}_{1}$} \\
\hline & $\mathrm{L}$ & $\mathrm{N}$ & $\mathrm{L}$ & $\mathrm{N}$ & $\mathrm{L}$ & $\mathrm{N}$ & $\mathrm{L}$ & $\mathrm{N}$ \\
\hline $\mathrm{P}_{1}$ & -0.07 & 0.29 & 0.22 & 0.16 & $-0.81^{*}$ & $-1.14^{*}$ & $-1.45^{* *}$ & $-2.81^{* * *}$ \\
\hline $\mathrm{P}_{2}$ & $0.33^{*}$ & $0.79^{* * *}$ & $0.42^{*}$ & $1.69^{* *}$ & $-2.37^{* *}$ & $-4.07^{* *}$ & $-1.35^{* * *}$ & $-2.68^{* * *}$ \\
\hline $\mathrm{P}_{3}$ & $-1.53^{* *}$ & $-0.64^{* *}$ & $-1.78^{* *}$ & $-0.91^{* *}$ & $2.23^{* *}$ & $1.23^{*}$ & $1.99^{* *}$ & $1.83^{* *}$ \\
\hline $\mathrm{P}_{4}$ & $-1.07^{* *}$ & $-0.97^{* *}$ & $-1.12^{* *}$ & $-1.21^{* *}$ & $-3.48^{* *}$ & $-5.22^{* *}$ & $-4.00^{* * *}$ & $-4.35^{* * *}$ \\
\hline $\mathrm{P}_{5}$ & $1.20^{* *}$ & $1.29^{* *}$ & $0.95^{* *}$ & $1.23^{* *}$ & $2.85^{* *}$ & $3.07^{* *}$ & $2.44^{* *}$ & $3.39^{* *}$ \\
\hline $\mathrm{P}_{6}$ & $-0.53^{* *}$ & $-0.61^{* *}$ & -0.12 & -0.21 & $-0.84^{*}$ & 0.18 & -0.19 & -0.34 \\
\hline $\mathrm{P}_{7}$ & $1.60^{* *}$ & 0.36 & $1.75^{* *}$ & 0.26 & 0.33 & $4.20^{* *}$ & $1.68^{* *}$ & $3.94^{* *}$ \\
\hline $\mathrm{P}_{8}$ & 0.07 & $-0.51^{* *}$ & -0.32 & $-1.01^{* *}$ & $2.09^{* *}$ & $1.75^{* *}$ & $0.88^{* *}$ & $1.04^{*}$ \\
\hline $\mathrm{P}_{1} \times \mathrm{P}_{2}$ & -1.08 & $-2.98^{* *}$ & $-2.61^{* *}$ & $-1.90^{* *}$ & -0.91 & 1.35 & -0.36 & 0.81 \\
\hline $\mathrm{P}_{1} \times \mathrm{P}_{3}$ & $-1.21^{*}$ & $-2.88^{* *}$ & -1.08 & $-1.30^{*}$ & $7.58^{* *}$ & 1.93 & 1.25 & -0.34 \\
\hline $\mathrm{P}_{1} \times \mathrm{P}_{4}$ & -1.01 & $-1.88^{* *}$ & $-1.75^{* *}$ & 0.00 & $-2.79^{* *}$ & -1.55 & -0.59 & 0.49 \\
\hline $\mathrm{P}_{1} \times \mathrm{P}_{5}$ & $-3.28^{* *}$ & 0.85 & $-2.48^{* *}$ & $-1.43^{*}$ & $6.36^{* *}$ & 1.10 & 0.18 & 0.52 \\
\hline $\mathrm{P}_{1} \times \mathrm{P}_{6}$ & $-2.21^{* *}$ & $-4.25^{* *}$ & -0.08 & $-3.66^{* *}$ & -0.20 & 1.78 & -0.34 & -0.44 \\
\hline $\mathrm{P}_{1} \times \mathrm{P}_{7}$ & $-3.01^{* *}$ & $-3.21^{* *}$ & $-1.95^{* *}$ & -0.13 & $-4.23^{* *}$ & -1.66 & $-3.76^{* * *}$ & -2.81 \\
\hline $\mathrm{P}_{1} \times \mathrm{P}_{8}$ & $-2.15^{* *}$ & $-3.35^{* *}$ & $-1.88^{* *}$ & -0.86 & -1.45 & 0.23 & 0.39 & -2.84 \\
\hline $\mathrm{P}_{2} \times \mathrm{P}_{3}$ & $-1.61^{* *}$ & $-2.71^{* *}$ & $-1.95^{* *}$ & $-1.83^{* *}$ & -1.70 & 2.51 & -0.29 & 0.66 \\
\hline $\mathrm{P}_{2} \times \mathrm{P}_{4}$ & $-1.41^{*}$ & $-1.38^{*}$ & -0.61 & -0.53 & -1.81 & -0.35 & 0.63 & $3.29^{* *}$ \\
\hline $\mathrm{P}_{2} \times \mathrm{P}_{5}$ & $-1.68^{* *}$ & $-3.98^{* *}$ & $-1.35^{*}$ & 0.04 & $-2.38^{*}$ & -0.39 & -0.01 & 2.04 \\
\hline $\mathrm{P}_{2} \times \mathrm{P}_{6}$ & $-1.28^{*}$ & $-3.75^{* *}$ & -0.28 & -0.53 & -1.49 & $3.34^{*}$ & -1.07 & 2.03 \\
\hline $\mathrm{P}_{2} \times \mathrm{P}_{7}$ & $-3.41^{* *}$ & $-3.71^{* *}$ & $-2.15^{* *}$ & $-1.66^{*}$ & $-2.25^{*}$ & -0.88 & $\begin{array}{l}-0.80 \\
\end{array}$ & 0.24 \\
\hline $\mathrm{P}_{2} \times \mathrm{P}_{8}$ & $-1.21^{*}$ & $-1.85^{* *}$ & $-1.41^{*}$ & $-1.40^{*}$ & $7.99^{* *}$ & $5.01^{* *}$ & $2.58^{* *}$ & $6.01^{* *}$ \\
\hline $\mathrm{P}_{3} \times \mathrm{P}_{4}$ & 0.45 & $-1.61^{* *}$ & -0.41 & $2.07^{* *}$ & $9.78^{* * *}$ & $5.49^{* *}$ & 1.13 & 0.93 \\
\hline $\mathrm{P}_{3} \times \mathrm{P}_{5}$ & $-1.15^{*}$ & $-2.88^{* * *}$ & $-1.15^{*}$ & $-1.36^{*}$ & $3.35^{* *}$ & -0.36 & 0.19 & -0.87 \\
\hline $\mathrm{P}_{3} \times \mathrm{P}_{6}$ & -0.08 & $-1.31^{*}$ & $1.92^{* *}$ & -1.26 & $-2.73^{*}$ & -1.10 & 1.28 & 1.43 \\
\hline $\mathrm{P}_{3} \times \mathrm{P}_{7}$ & -0.21 & -0.61 & 0.05 & 0.27 & -2.16 & 0.29 & -0.05 & $5.19^{* *}$ \\
\hline $\mathrm{P}_{3} \times \mathrm{P}_{8}$ & -0.01 & $-1.41^{*}$ & $-1.21^{*}$ & $-2.13^{* *}$ & $-3.69^{* *}$ & -1.36 & 0.96 & 1.72 \\
\hline $\mathrm{P}_{4} \times \mathrm{P}_{5}$ & 0.39 & $-2.21^{* *}$ & 0.19 & 0.27 & 0.56 & 1.65 & 0.66 & 0.86 \\
\hline $\mathrm{P}_{4} \times \mathrm{P}_{6}$ & $1.45^{*}$ & $-1.31^{*}$ & $1.25^{*}$ & 0.37 & 0.53 & 0.76 & -1.27 & 2.48 \\
\hline $\mathrm{P}_{4} \times \mathrm{P}_{7}$ & $-2.01^{* *}$ & $-1.95^{* *}$ & $-1.28^{*}$ & $-3.43^{* *}$ & -0.88 & 1.15 & -0.17 & 0.71 \\
\hline $\mathrm{P}_{4} \times \mathrm{P}_{8}$ & -0.48 & -1.08 & 0.79 & $-2.16^{* *}$ & -0.84 & -1.57 & $-2.93^{* *}$ & -0.76 \\
\hline $\mathrm{P}_{5} \times \mathrm{P}_{6}$ & -0.15 & $-2.25^{* *}$ & -0.81 & $-1.40^{*}$ & 1.21 & 1.22 & -1.59 & 0.05 \\
\hline $\mathrm{P}_{5} \times \mathrm{P}_{7}$ & $-2.28^{* *}$ & $-4.21^{* *}$ & $-2.68^{* *}$ & $-3.86^{* *}$ & -0.12 & 0.99 & -0.37 & 0.33 \\
\hline $\mathrm{P}_{5} \times \mathrm{P}_{8}$ & -0.75 & $-2.35^{* *}$ & -0.61 & -0.60 & 0.35 & 1.94 & -0.54 & $3.45^{*}$ \\
\hline $\mathrm{P}_{6} \times \mathrm{P}_{7}$ & -0.55 & $-1.65^{* *}$ & $-1.61^{* *}$ & 0.24 & 0.68 & 2.54 & $-2.63^{* * *}$ & 1.87 \\
\hline $\mathrm{P}_{6} \times \mathrm{P}_{8}$ & 0.99 & -0.78 & 0.45 & $1.50^{*}$ & 1.22 & 2.38 & -1.76 & 0.33 \\
\hline $\mathrm{P}_{7} \times \mathrm{P}_{8}$ & $-1.15^{*}$ & $-1.41^{* *}$ & $-1.41^{*}$ & 0.37 & -1.54 & $3.77^{*}$ & $-2.32^{*}$ & -1.43 \\
\hline S.E. gi & 0.17 & 0.20 & 0.20 & 0.22 & 0.37 & 0.51 & 0.32 & 0.49 \\
\hline S.E. sij & 0.57 & 0.58 & 0.57 & 0.66 & 1.13 & 1.56 & 0.98 & 1.51 \\
\hline S.E. (gi-gj) & 0.28 & 0.28 & 0.28 & 0.33 & 0.56 & 0.77 & 0.48 & 0.74 \\
\hline S.E. (sij-sik) & 0.84 & 0.87 & 0.85 & 0.98 & 1.67 & 2.30 & 1.45 & 2.23 \\
\hline
\end{tabular}

*and **: significant at 0.05 and 0.01 levels of probability, respectively. 
Table 7. General (GCA) and specific (SCA) combining ability effects for 100-grain weight and grain yield/plant of the parents and their $\mathrm{F}_{1}$ 's and $\mathrm{F}_{2}$-populations under normal $(\mathrm{N})$ and late (L) planting dates during 2017/2018 season.

\begin{tabular}{|c|c|c|c|c|c|c|c|c|}
\hline \multirow[t]{3}{*}{ Genotypes } & \multicolumn{4}{|c|}{ 100-grain weight (g) } & \multicolumn{4}{|c|}{ Grain yield/plant (g) } \\
\hline & \multicolumn{2}{|c|}{$\mathrm{F}_{2}$} & \multicolumn{2}{|c|}{$\mathrm{F}_{1}$} & \multicolumn{2}{|c|}{$\mathrm{F}_{2}$} & \multicolumn{2}{|c|}{$\mathrm{F}_{1}$} \\
\hline & $\mathrm{L}$ & $\mathrm{N}$ & $\mathrm{L}$ & $\mathrm{N}$ & $\mathrm{L}$ & $\mathrm{N}$ & $\mathrm{L}$ & $\mathrm{N}$ \\
\hline $\mathrm{P}_{1}$ & 0.05 & $-0.22^{* *}$ & $-0.25^{* *}$ & $-0.14^{*}$ & $-0.65^{* *}$ & 0.41 & -0.29 & 0.27 \\
\hline $\mathrm{P}_{2}$ & $0.06^{*}$ & $0.20^{\text {*** }}$ & $0.24^{* *}$ & $0.42^{* *}$ & $0.66^{* *}$ & $0.61^{* *}$ & $1.24^{* *}$ & $1.67^{* * *}$ \\
\hline $\mathrm{P}_{3}$ & $0.25^{* *}$ & $0.51^{* *}$ & $0.29^{* *}$ & $0.16^{* *}$ & 0.14 & $-0.67^{* *}$ & -0.02 & $-2.23^{* * *}$ \\
\hline $\mathrm{P}_{4}$ & $-0.09^{* *}$ & -0.08 & $-0.20^{* *}$ & -0.08 & $-0.73^{* *}$ & $-0.52^{* *}$ & $-0.78^{* *}$ & -0.16 \\
\hline $\mathrm{P}_{5}$ & $-0.13^{* *}$ & $-0.22^{* *}$ & 0.02 & -0.03 & -0.26 & 0.14 & -0.17 & 0.22 \\
\hline $\mathrm{P}_{6}$ & $-0.08^{* *}$ & $-0.26^{* *}$ & $-0.15^{* *}$ & $-0.25^{* *}$ & -0.39 & -0.08 & -0.23 & 0.17 \\
\hline $\mathrm{P}_{7}$ & -0.03 & 0.04 & $0.16^{* *}$ & 0.02 & $1.27^{* * *}$ & 0.28 & 0.44 & 0.52 \\
\hline $\mathrm{P}_{8}$ & -0.02 & 0.04 & $-0.12^{* *}$ & -0.10 & -0.03 & -0.17 & -0.19 & -0.45 \\
\hline $\mathrm{P}_{1} \times \mathrm{P}_{2}$ & $-0.47^{* *}$ & -0.30 & $-0.35^{*}$ & $-0.72^{* *}$ & 0.36 & 0.04 & 0.49 & -0.31 \\
\hline $\mathrm{P}_{1} \times \mathrm{P}_{3}$ & $0.97^{* *}$ & $0.66^{* *}$ & $0.62^{* * *}$ & $0.65^{* *}$ & -0.07 & $-1.70^{*}$ & 0.42 & -2.01 \\
\hline $\mathrm{P}_{1} \times \mathrm{P}_{4}$ & $0.14^{* *}$ & -0.03 & 0.16 & $0.59^{* *}$ & $-1.27^{*}$ & -0.26 & 0.27 & 1.33 \\
\hline $\mathrm{P}_{1} \times \mathrm{P}_{5}$ & $0.32^{* *}$ & 0.00 & 0.26 & $0.55^{* *}$ & 0.04 & $-5.65^{* *}$ & $2.16^{* *}$ & 2.08 \\
\hline $\mathrm{P}_{1} \times \mathrm{P}_{6}$ & $0.19^{* *}$ & 0.17 & 0.21 & $0.59^{* *}$ & 0.50 & $-1.34^{*}$ & 1.01 & -0.01 \\
\hline $\mathrm{P}_{1} \times \mathrm{P}_{7}$ & 0.04 & 0.01 & 0.14 & 0.30 & -1.26 & 0.48 & 0.20 & -1.29 \\
\hline $\mathrm{P}_{1} \times \mathrm{P}_{8}$ & $0.07^{*}$ & $-0.44^{* *}$ & 0.15 & 0.22 & 0.39 & 0.42 & $-1.68^{*}$ & -2.22 \\
\hline $\mathrm{P}_{2} \times \mathrm{P}_{3}$ & $-0.17^{* *}$ & 1.12 & $-0.27^{*}$ & $-0.38^{*}$ & 0.91 & -0.56 & 1.57 & -1.46 \\
\hline $\mathrm{P}_{2} \times \mathrm{P}_{4}$ & $0.18^{* *}$ & $0.83^{* *}$ & $0.64^{* *}$ & $0.92^{* *}$ & -0.29 & 0.01 & $2.50^{* *}$ & $-4.05^{* * *}$ \\
\hline $\mathrm{P}_{2} \times \mathrm{P}_{5}$ & $0.29^{* *}$ & $-0.40^{*}$ & 0.11 & $0.43^{*}$ & $1.85^{* *}$ & 0.31 & -0.55 & $-3.80^{*}$ \\
\hline $\mathrm{P}_{2} \times \mathrm{P}_{6}$ & $0.22^{* *}$ & -0.08 & $0.36^{* *}$ & -0.17 & -0.08 & $-2.28^{* *}$ & $\begin{array}{l}-0.64 \\
\end{array}$ & -0.18 \\
\hline $\mathrm{P}_{2} \times \mathrm{P}_{7}$ & $0.63^{* *}$ & -0.20 & $-0.34^{*}$ & $1.11^{* *}$ & 0.29 & $-2.87^{* *}$ & -1.43 & $4.57^{* * *}$ \\
\hline $\mathrm{P}_{2} \times \mathrm{P}_{8}$ & $0.64^{* *}$ & $0.45^{* *}$ & $0.37^{* * *}$ & $0.65^{* *}$ & $3.67^{* *}$ & $2.66^{* *}$ & $2.68^{* *}$ & $4.57^{* *}$ \\
\hline $\mathrm{P}_{3} \times \mathrm{P}_{4}$ & $-0.13^{* *}$ & $-0.86^{* *}$ & $-0.89^{* *}$ & $-0.45^{* *}$ & -0.90 & -0.07 & -1.06 & -0.61 \\
\hline $\mathrm{P}_{3} \times \mathrm{P}_{5}$ & $-0.67^{* *}$ & $-0.38^{*}$ & 0.07 & -0.12 & 0.79 & $2.19^{* *}$ & 0.75 & $4.26^{* *}$ \\
\hline $\mathrm{P}_{3} \times \mathrm{P}_{6}$ & $-0.70^{\text {** }}$ & -0.25 & $-0.35^{*}$ & -0.16 & 0.91 & 0.56 & -0.93 & 1.87 \\
\hline $\mathrm{P}_{3} \times \mathrm{P}_{7}$ & $0.45^{* *}$ & $0.35^{*}$ & -0.18 & -0.12 & $-1.83^{* *}$ & 0.63 & -0.28 & -0.86 \\
\hline $\mathrm{P}_{3} \times \mathrm{P}_{8}$ & $-0.77^{* *}$ & -0.61 & $-0.46^{* *}$ & $-0.82^{* *}$ & $-2.54^{* *}$ & $-1.63^{*}$ & -1.11 & -1.88 \\
\hline $\mathrm{P}_{4} \times \mathrm{P}_{5}$ & 0.04 & $-0.43^{* *}$ & -0.07 & $-0.45^{* *}$ & $-3.17^{* *}$ & -0.74 & $-3.59^{* * *}$ & $-3.66^{*}$ \\
\hline $\mathrm{P}_{4} \times \mathrm{P}_{6}$ & 0.03 & -0.21 & $0.53^{* *}$ & -0.09 & 0.87 & $2.87^{* *}$ & 1.56 & 1.84 \\
\hline $\mathrm{P}_{4} \times \mathrm{P}_{7}$ & $-0.37^{* *}$ & 0.07 & 0.16 & -0.21 & 0.19 & $-4.40^{* *}$ & -0.02 & $5.06^{* *}$ \\
\hline $\mathrm{P}_{4} \times \mathrm{P}_{8}$ & $-0.33^{* *}$ & -0.17 & 0.01 & -0.09 & $1.91^{* *}$ & $2.36^{* *}$ & $3.04^{* *}$ & $7.48^{* *}$ \\
\hline $\mathrm{P}_{5} \times \mathrm{P}_{6}$ & $0.22^{* *}$ & $-0.43^{* *}$ & $-0.41^{* *}$ & $-0.56^{* *}$ & $-2.14^{* *}$ & $-2.32^{* *}$ & $-2.76^{* *}$ & -2.49 \\
\hline $\mathrm{P}_{5} \times \mathrm{P}_{7}$ & $-0.09^{* *}$ & 0.25 & -0.12 & $-0.50^{* *}$ & -1.25 & $-1.83^{* *}$ & $4.06^{* *}$ & 0.47 \\
\hline $\mathrm{P}_{5} \times \mathrm{P}_{8}$ & $0.50^{* *}$ & $0.34^{*}$ & -0.10 & $0.74^{* *}$ & 0.29 & -0.42 & -0.56 & 1.15 \\
\hline $\mathrm{P}_{6} \times \mathrm{P}_{7}$ & 0.01 & $-0.34^{*}$ & -0.13 & $-0.36^{*}$ & -0.44 & -0.08 & -0.04 & $6.89^{* *}$ \\
\hline $\mathrm{P}_{6} \times \mathrm{P}_{8}$ & $-0.28^{* *}$ & -0.20 & 0.09 & 0.03 & $-3.14^{* *}$ & $3.11^{* *}$ & 0.25 & $4.82^{* * *}$ \\
\hline $\mathrm{P}_{7} \times \mathrm{P}_{8}$ & $0.84^{* *}$ & $-0.41^{*}$ & 0.01 & $-0.55^{* *}$ & 0.44 & $2.28^{* *}$ & -0.37 & $-5.18^{* * *}$ \\
\hline S.E. gi & 0.03 & 0.06 & 0.04 & 0.06 & 0.22 & 0.22 & 0.28 & 0.49 \\
\hline S.E. $s i j$ & 0.10 & 0.17 & 0.14 & 0.17 & 0.65 & 0.68 & 0.84 & 1.49 \\
\hline S.E. (gi-gj) & 0.05 & 0.1 & 0.07 & 0.10 & 0.32 & 0.33 & 0.41 & 0.73 \\
\hline S.E. (sij-sik) & 0.14 & 0.26 & 0.20 & 0.57 & 0.96 & 1.00 & 1.24 & 0.57 \\
\hline
\end{tabular}

*and **: significant at 0.05 and 0.01 levels of probability, respectively. 


\section{References}

Abdel-Nour, Nadya, Manal A. R. and Hassan A. (2009) 'Determination of gene effects and variance in three bread wheat crosses for low water (drought), Egypt. J. Plant Breed., 13, pp. 235-249.

Ahmed, A. A. S. (2003) 'Diallel analysis and stability of yield and yield components in bread wheat (Triticum astivum L. em. Thell)', M.Sc. Thesis, Fac. of Agric. Assiut Univ., Egypt.

Ahmed, M. S. H. and Mohamed S. M. S. (2009) 'Diallel crosses of bread wheat (Triticum aestivum L.) at two sowing dates 1 . Genetic analysis of yield and its components', Egypt. J. Plant Breed. 13, pp. 281-301.

Akinci, C. (2009) 'Heterosis and combining ability estimates in $6 \times 6$ half-diallel crosses of durum wheat (Triticum astivum Desf)', Bulg. J. Agric., 15, pp. 214 - 221.

Ali, M. A. (2006) 'Combining ability and stability analysis in spring wheat under four level of soil available water', Assiut J. Agric. Sci., 37(2), pp. 95 - 118.

Ankita, S., Anil, K., Ekhlaque, A., Swati and Jaiswal, J. p. (2012) ' Combining ability and gene action studies for grain yield, its components and quality traits in bread wheat ( $T$. astivum L. em. Thell.)', Electronic J. Plant Breeding, 3(4), pp. $964-972$.

Ashoush, H. A., Hamada A. A. and Darwish I. H. (2001) 'Heterosis and combining ability in $F_{1}$ and $F_{2}$ diallel crosses of wheat (Triticum aestivum L. em. Thell)', Mansoura J. Agric. Res. 26(5),pp. 2579-2592.

Asmaa M. Badr; Ahmed M.F., Esmail A.M. and Rashed M.A. (2018) 'Heat tolerance in some bread wheat genotypes under two sowing dates', Arab Univ. J. Agric. Sci., Ain Shams
Univ., Cairo Special Issue, 26(2A), pp. 987-1000.

Bajaniga N., Pansuriga A. G., Vekaria D. M., Singh C. and Savaliga J.J. (2019) 'Combining ability for grain yield and its components in durum wheat (Triticum durum Desf. )', Ind. J. Pure App. Biosci. 7, pp. 217-224.

Darwish, I. H. I.; El-Sayed E. and Waffa, El-Awady (2006) 'Genetical studies of heading date and some agronomic characters in wheat', Annals of Agric. Sci. Moshtohor., 44(2),pp. 427-452.

Dencic, S., R. Kastori, Kobiljski B. and Duggan B. (2000) 'Evaluation of grain yield and its components in wheat cultivars and landraces under near optimal and drought conditions', Euphytica 113, pp. 43 - 52.

Dueby, L. K., Sastry E. V. D. and Sinha K. (2001) 'Heterosis for yield and yield components in bread wheat ((Triticum aestivum L.) under saline and normal environments ', Annals of Arid Zone, 40, pp. $57-60$.

El-Awady, Wafaa A. 2011 'Analysis of yield and its components using five parameters for three bread wheat crosses' Egyt. J. Agric. Res., 89(3); pp. $993-1003$.

El-Karamity, A. S., Salem M. A., Tamam A. M. and Mohamed M. I. (2007) 'Genetical studies on grain yield and its components in durum wheat (Triticum durum var. durum)', Minia J. of Agric. Res. 5(27), pp. 967-985.

El-Sayed, E. M. A. (2004) 'A diallel cross analysis for some quantitative characters in bread wheat (Triticum aestivum L.)', Egypt J. Agric. Res., 83 (4), pp. 1665-1675.

Ezatollah, F., Rafiee F. and Hasheminasab H. (2013) 'valuation of genetic parameters of agronomic and morpho- 
physiological indicators of drought tolerance in bread wheat (Triticum aestivum L.) using diallel mating design', Australina J. of Crop Sci., 7 (2), pp. $268-275$

Falconer, D.S. (1990) 'Selection in different environment: effects on environmental sensitivity (reaction norm) and mean performance,' Genrt. Res. Camb., 56, pp. 57-70.

Farhad, A., Khan S., Ahmad S. Q., Khan H., Khan A. and Muhammad F. (2011) 'Genetic analysis of some quantitative traits in bread wheat across environments', African J. of Agri. Res., 6 (3), pp. 686-692.

Fisher, R. A. and Maurer R. O. (1978) 'Drought resistance in spring wheat cultivars. 1- Grain yield responses', Aust. J. Agric. Res.; 29, pp. 897-912.

Griffing, B. (1956) 'Concept of general and specific combining ability in relation to diallel crossing systems', Aust. J. Biol . Sci., 9, pp. 463-493.

Hamada, A. A. and Tawfelis M. B.. (2001)'Genetic and graphical analysis of diallel crosses of some bread wheat (Triticum aestivum L. em. Thell)', J. Agric. Res. Tanta Univ. 27(4), pp. 633647.

Hassan, M. O. M. (2015) 'Combining ability and genetic components in durum wheat under heat stress conditions in reclaimed soils', M.Sc. Thesis, Fac. of Agric. Assiut Univ., Egypt.

Hassan, M. I. (2016) 'Assessment of genetic diversity in bread wheat genotypes based on heat tolerance and SSR markers', Assiut J. Agric. Sci. 47 (5), pp. 37-55.

Hassan M.I., Mohamed E.A., El-Rawy M.A. and Amein K.A. (2016) 'Evaluating interspecific wheat hybrids based on heat and drought stress tolerance', J. Crop Sci. Biotech. 19 (1), pp. 85-98.

Hoffman, B. and Burucs Z. (2005) Adaptation of wheat (Triticum astivum L.) genotypes and related species to water deficiency', Cereal Research Comm., 33, pp. $681-687$.

Hayman, B.I. 91954a) 'The theory of analysis of diallel crosses II', Genetics, 43, pp. 789-809.

Hayman, B.I. (1954b) 'The analysis of variance of diallel tables', Biometrics, 10, pp. 235-244.

Iqbal, M. Raja, Yasmeen N.I., Hussain F., Ejaz M., Shah M., M.A. (2017) 'Impacts of heat stress on wheat: A critical review', Advances in Crop Science and Technology, 5, pp. 251.

Jinks, J. L. (1954) 'The analysis of continuous variation in a diallel cross of Nicotianarustica varieties', Genetics, 39, pp. 767-788.

Kapoor, E., Mandal S. K. and Dey T. (2011) 'Combing ability analysis for yield and yield attributing traits in winter and spring wheat combinations', J. Wheat Res., 3(1), pp. $52-58$.

Khalid, M. A. and Abd EL-dayem S. M. (2014) 'Combing ability analysis for grain yield and its attributes in bread wheat under stress and normal irrigation conditions', J. Plant Production, Mansoura univ., Vol. 5, pp. 255-266.

Khierralla, K. A., El-Morshidy M. A. and Zakaria M. M. (2001) 'Inheritance of earliness and yield in bread wheat under favourable and late planting dates', The Second PI. Breed. Conf., October 2, 2001 assiut Univ, pp. 219 ; 239.

Mohamed, M. M. (2007) ' Genetic studies in durum wheat breeding for heat tolerance', M.Sc. Thesis, Fac. of Agric. El-Minia Univ., Egypt. 
Moussa, A. M. (2010) 'Estimation of epistasis additive and dominance variation in certain bread wheat (Triticum astivum L.) crosses', J. Plant Biometrical Approaches. $2^{\text {nd }}$ McGrawHill Book Company, New York, London.

Nagwa, Salem R. A. (2007) 'Heterosis and combining ability for grain yield components of some durum wheat crosses,' Egypt J. Agric. Res., 85(2), pp. $563-571$.

Nassar, S. M. A. M. (2013) 'Genetic variance and combining ability in bread wheat under drought stress conditions', Ph.D. Thesis, Fac. of Agric. Assiut Univ., Egypt.

Jaiswal B., Prasad S., Dwivedi R., Singh S., Rani R., Shrivastava S., Kumar A. and Yadav R.K. (2017) 'tudy of Yield and Yield Components of Wheat (Triticum aestivum L.) Genotypes at Grain Filling Stage under Heat Regimes', Int. J. Pure App. Biosci. 5(4), pp. 331-340.

Kumar, J, Singh S. K., Singh L., Kumar M., Srivastava M., Singh J. and Kumar A. (2017) 'Combining ability analysis for yield and its components in bread wheat (Triticum aestivum L.) under biotic stress', Int. J. Curr. Microbiol. App. Sci.6, pp. 24-39.

Kumar, S., Singh S. K., Gupta S. K., Vishwanath, Kumar P. Y. S., Kumar J., Bind H. N. and Singh L. (2017) 'ombining ability in relation to wheat (triticum aestivum L.) breeding programme under heat stress environment', Int. J. Curr. Microbiology and Applied. Sci.6, pp. 3065-3073.

Nayeem, K. A. and Veer M. V. (2000) 'Combining ability for heat tolerance traits in bread wheat (Triticum aestivum L.)', Indian J. Genet. 60(3),pp. 287-295.
Samra, J. S., Dhilion S. S. and Kahlon P. S. (1989) 'Response of wheat varieties to date of sowing', Indian J. Agron. 34,pp. $286-289$.

Sharma Y., Dodiga N. S., Dubey R. B. and Khn R.. (2019) 'Combining ability analysis in bread wheat (triticum aestivum (L.) EM. Thell) under different Environmental conditions', Bangladesh J. Bot. 48, pp. 89-93.

Snedecor, G. W. and Cochran W. G. (1967) ' Statistical Methods', 6th Ed., Iowa State Univ. press, Ames, Iowa, U.S.A.

Taghian, A. S. and Abo-Elwafa A.. (2003) 'Multivariate and RAPD analysis of drought tolerance in spring wheat ( Triticum aestivum L)', Assiut J. of Agric. Sci., 34(5), pp. 1-25.

Wajid, A. J., Baloch M. J., Kumbhar M. B., Khan N. U. and Kerio M. I. (2011) ' Effect of water stress on physiological and yield parameters at anthesis stage in elite spring wheat cultivars', Sarhad J. Agric., 27 (1), pp. 59-65.

Wahid, A., S. Gelani, Ashraf M. and Foolad M. (2007) 'Heat tolerance in plant', An overview. Environ. Exp. Bot., 61, pp. $199-224$.

Yao, J. B., Ma H. X., Ren L. J., Zhang P. P., Yang X. M., Yao G. C., Zhang P. and Zhon M. P. (2011) 'Genetic analysis of plant height and its components in diallel crosses of bread wheat ( Triticum aestivum L) '., Australina J. of Crop Sci., 5(11), pp. 1408 - 1418.

Zakaria, M. M. (1999) 'Genetical and agronomic studies on heat tolerance and yield in wheat (Triticum aestivum L.)', M.Sc. Thesis, Fac. of Agric., Assiut Univ. 